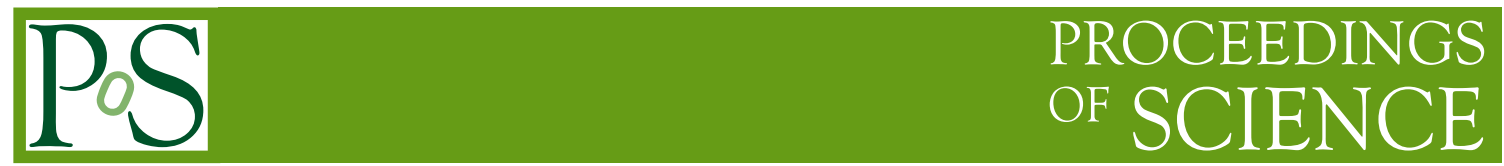

\title{
Gravitational wave astronomy with compact binary mergers
}

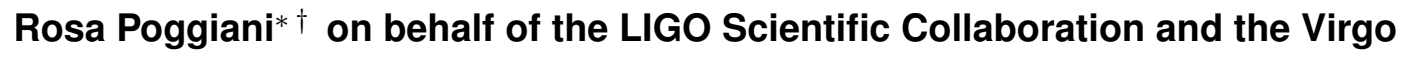 \\ Collaboration \\ Università di Pisa and Istituto Nazionale di Fisica Nucleare, Sezione di Pisa \\ E-mail: rosa.poggiani@unipi.it
}

\begin{abstract}
The detection of gravitational waves from the mergers of binary black holes and binary neutron stars has opened a new observational window in astronomy. This paper presents a review of the events detected during the first runs of Advanced LIGO and Advanced Virgo and their impact on astrophysics and cosmology.
\end{abstract}

The Golden Age of Cataclysmic Variables and Related Objects V(GOLDEN2019)

2-7 September 2019

Palermo, Italy

${ }^{*}$ Speaker.
${ }^{\dagger}$ Corresponding author

(C) Copyright owned by the author(s) under the terms of the Creative Commons 


\section{Introduction}

The first two observing runs, $\mathrm{O} 1$ and $\mathrm{O} 2$, of Advanced LIGO, joined by Advanced Virgo during $\mathrm{O} 2$, have produced the first direct detection of gravitational waves from the merger of stellar mass black holes, GW150914 [2], and the first detection of a binary neutron star merger, GW170817 [19]. In addition, other binary black hole mergers have been detected: GW151226 [15], GW170104 [16], GW170608 [17], GW170814 [18] (the first detection with three interferometers), GW151012, GW170729, GW170809, GW170818 and GW170823 [31]. All detections are part of GWTC-1, the First Gravitational Wave Transient Catalog [31], that will be discussed below. This paper reviews the detections of the GWTC-1 catalog, starting with GW150914, and their impact on astrophysics and cosmology; the paper also discusses some events of the observing run $\mathrm{O} 3$, that was ongoing at the time of conference.

\section{GW150914: The First Detection}

The event GW150914 is the first direct detection of gravitational waves and the first observation of a binary black hole merger [2]. On September 14, 2015 at 09:50:45 UTC the two Advanced LIGO interferometers observed a transient signal that swept in frequency from 35 to 250 $\mathrm{Hz}$, achieving a peak gravitational wave strain of $1.0 \times 10^{-21}$ (Fig. 1).

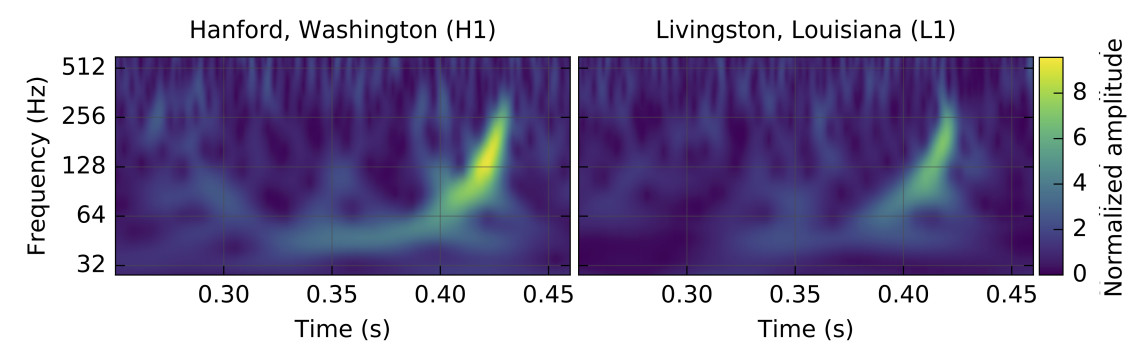

Figure 1: Time-frequency maps of GW150914 observed by the LIGO Hanford (H1, left panel) and LIGO Livingston (L1, right panel) interferometers. Adapted from [2].

The signal was consistent with the General Relativity waveform for the inspiral and the merger of two black holes and the ringdown of the final black hole. The signal had a signal-to-noise ratio of 24 and a false alarm rate smaller than 1 event per 203000 years, with a significance larger than $5.1 \sigma$ [2]. The luminosity distance of GW150914 was $410_{-180}^{+160} \mathrm{Mpc}$ [2]. The masses of the initial black holes were $36_{-4}^{+6} \mathrm{M}_{\odot}$ and $29_{-4}^{+4} \mathrm{M}_{\odot}$, while the final black hole had a mass of $62_{-4}^{+4} \mathrm{M}_{\odot}$ and a spin of $0.67_{-0.07}^{+0.05}$ [2]. About $3.0_{-0.5}^{+0.5} \mathrm{M}_{\odot} \mathrm{c}^{2}$ were emitted as gravitational waves [2]. GW150914 was localized within an annulus region of $610 \mathrm{deg}^{2}$ [4], [12].

The first direct detection of gravitational waves demonstrated the potential of gravitational astronomy. The GW150914 observation has several astrophysical implications [11]. Binary black hole mergers were predicted in formation models involving isolated binaries in galactic fields [236] or dynamical interactions in dense stellar environments [216]. Population studies had predicted that binary black hole mergers could be the dominant source from the point of view of the detection rates [161]. The observation of GW150914 showed that heavy stellar mass black holes, with masses 
above $25 \mathrm{M}_{\odot}$, can form [11]. GW150914 was formed in an environment with a metallicity lower than about one half of the solar metallicity [11]. The GW150914 event constrained the rate of stellar mass binary black hole mergers in the range 2-600 $\mathrm{Gpc}^{-3} \mathrm{yr}^{-1}$ [2], [14]. GW150914 has been used for different tests of General Relativity [3]. The mass and spin of the final black hole estimated from the inspiral and post-inspiral stages are consistent with a binary black hole merger, while the after peak stage is consistent with a damped quasinormal mode of a black hole [3]. The detection of GW150914 constrained the mass of the graviton to be smaller than $1.2 \times 10^{-22} \mathrm{eV} / \mathrm{c}^{2}$ [2], [3]. The observation of GW150914 allowed to constrain the energy density of the stochastic background from binary black holes [5]. Further analyses of the GW150914 merger are described in [6], [7], [8], [9].

\section{The GWTC-1 Catalog}

The First Gravitational Wave Transient Catalog, GWTC-1, reports 11 detections of gravitational waves from compact binary mergers and a compilation of other less significant triggers during the $\mathrm{O} 1, \mathrm{O} 2$ observing runs [31]. The candidate events were selected by three search pipelines; two matched filtering searches using relativistic waveform models, PyCBC [189], [237] and GstLAL [206], [173], and one unmodeled search for excess noise from short duration bursts, coherent WaveBurst (cWB) [150].

The searches identified 11 confident detections, ten binary black mergers and one binary neutron star merger, among them some unpublished events. The parameters of the previously published events were updated with the new analysis. The initial threshold for event selection was a False Alarm Rate (FAR) of 1 per 30 days in at least one of the matched filter searches, assigning the $G W$ designation to events whose probability of astrophysical origin in either matched filter search is above $50 \%$ and the marginal event designation to the others. During run O1, two confident detections of binary black hole merger were made, GW150914 [2] and GW151226 [15]. A third candidate recovered in the reanalysis, GW151012 (formerly LVT151012), is consistent with an astrophysical binary black hole merger. During run O2, three binary black hole mergers had been published, GW170104 [16], GW170608 [17], and GW170814 [18], and one binary neutron star merger, GW170817 [19]. The new analysis recovered additional binary black hole mergers: GW170729, GW170809, GW170818 and GW170823 [31]. The relevant source parameters for the GWTC-1 detections are reported in Table 1.

For binary black hole mergers, the posterior distributions are from an effective precessing spin waveform model (IMRPhenomPv2) [136], [147], [140] and a fully precessing model (SEOBNRv3) [228], [195], [67]. For GW170817, the posterior distributions are from the time domain models SEOBNRv4T [137] and TEOBResumS [75], [184] and the frequency domain models SEOBNRv4NRT [201], [82] [105], [106], IMRPhenomPv2NRT [136], [147], [140], [105], [106], TaylorF2 [208], [76], [98], [77], [78], [175], [65], [246], [99], [100], [80], [79], [81], [177].

The signal of a binary black hole merger depends on 8 intrinsic parameters: the masses $\mathrm{m}_{1}$, $\mathrm{m}_{2}$ and the two spin vectors $\vec{S}_{i}$, with three components each. In addition, 7 extrinsic parameters are needed: the sky position (right ascension and declination), the orbital inclination $i$, the luminosity distance $\mathrm{d}_{L}$, the polarization angle $\psi$, and the time $\mathrm{t}_{c}$ and phase $\phi_{c}$ of coalescence. The dimensionless spin vectors and spin magnitudes are defined against the maximum spin a Kerr black hole of 


\begin{tabular}{|c|c|c|c|c|c|c|c|c|c|c|c|}
\hline Event & $\mathrm{m}_{1}\left(\mathrm{M}_{\odot}\right)$ & $\mathrm{m}_{2}\left(\mathrm{M}_{\odot}\right)$ & $\mathrm{M}_{c}\left(\mathrm{M}_{\odot}\right)$ & $\chi_{e f f}$ & $\mathbf{M}_{f}\left(\mathbf{M}_{\odot}\right)$ & $\mathrm{a}_{f}$ & $\mathrm{E}_{r}\left(\mathrm{M}_{\odot} c^{2}\right)$ & $1_{\text {peak }}\left(\mathrm{erg} \mathrm{s}^{-1}\right)$ & $\mathrm{d}_{L}(\mathrm{Mpc})$ & $\mathrm{Z}$ & $\Delta \Omega\left(\operatorname{deg}^{2}\right)$ \\
\hline GW150914 & $35.6_{-3.1}^{+4.7}$ & $30.6_{-4.4}^{+3.0}$ & $28.6_{-1.5}^{+1.7}$ & $-0.01_{-0.13}^{+0.12}$ & $63.1_{-3.0}^{+3.4}$ & $0.69_{-0.04}^{+0.05}$ & $3.1_{-0.4}^{+0.4}$ & $3.6_{-0.4}^{+0.4} \times 10^{56}$ & $440_{-170}^{+150}$ & $0.09_{-0.03}^{+0.03}$ & 182 \\
\hline GW151012 & $23.2_{-5.5}^{+14.9}$ & $13.6_{-4.8}^{+4.1}$ & $15.2_{-1.2}^{+2.1}$ & $0.05_{-0.20}^{+0.31}$ & $35.6_{-3.8}^{+10.8}$ & $0.67_{-0.11}^{+0.13}$ & $1.6_{-0.5}^{+0.6}$ & $3.2_{-1.7}^{+0.8} \times 10^{56}$ & $1080_{-490}^{+550}$ & $0.21_{-0.09}^{+0.09}$ & 1523 \\
\hline GW151226 & $13.7_{-3.2}^{+8.8}$ & $7.7_{-2.5}^{+2.2}$ & $8.9_{-0.3}^{+0.3}$ & $0.18_{-0.12}^{+0.20}$ & $20.5_{-1.5}^{+6.4}$ & $0.74_{-0.05}^{+0.07}$ & $1.0_{-0.2}^{+0.1}$ & $3.4_{-1.7}^{+0.7} \times 10^{56}$ & $450_{-190}^{+180}$ & $0.09_{-0.04}^{+0.04}$ & 1033 \\
\hline GW170104 & $30.8_{-5.6}^{+7.3}$ & $20.0_{-4.6}^{+4.9}$ & $21.4_{-1.8}^{+2.2}$ & $-0.04_{-0.21}^{+0.17}$ & $48.9_{-4.0}^{+5.1}$ & $0.66_{-0.11}^{+0.08}$ & $2.2_{-0.5}^{+0.5}$ & $3.3_{-1.0}^{+0.6} \times 10^{56}$ & $990_{-430}^{+440}$ & $0.20_{-0.08}^{+0.08}$ & 921 \\
\hline GW170608 & $11.0_{-1.7}^{+5.5}$ & $7.6_{-2.2}^{+1.4}$ & $7.9_{-0.2}^{+0.2}$ & $0.03_{-0.07}^{+0.19}$ & $17.8_{-0.7}^{+3.4}$ & $0.69_{-0.04}^{+0.04}$ & $0.9_{-0.1}^{+0.0}$ & $3.5_{-1.3}^{+0.4} \times 10^{56}$ & $320_{-110}^{+120}$ & $0.07_{-0.02}^{+0.02}$ & 392 \\
\hline GW170729 & $50.2_{-10.2}^{+16.2}$ & $34.0_{-10.1}^{+9.1}$ & $35.4_{-4.8}^{+6.5}$ & $0.37_{-0.25}^{+0.21}$ & $79.5_{-10.2}^{+14.7}$ & $0.81_{-0.13}^{+0.07}$ & $4.8_{-1.7}^{+1.7}$ & $4.2_{-1.5}^{+0.9} \times 10^{56}$ & $2840_{-1360}^{+1400}$ & $0.49_{-0.21}^{+0.19}$ & 1041 \\
\hline GW & $35.0_{-5.9}^{+8.3}$ & $23.8_{-5.2}^{+5.1}$ & $24.9_{-1.7}^{+2.1}$ & $0.08_{-0.17}^{+0.17}$ & $56.3_{-3.8}^{+5.2}$ & $0.70_{-0.09}^{+0.08}$ & $2.7_{-0.6}^{+0.6}$ & $3.5_{-0.9}^{+0.6} \times 10^{56}$ & $1030_{-390}^{+320}$ & $0.20_{-0.07}^{+0.05}$ & 308 \\
\hline GW & $30.6_{-3.0}^{+5.6}$ & $25.2_{-4.0}^{+2.8}$ & $24.1_{-1.1}^{+1.4}$ & $0.07_{-0.12}^{+0.12}$ & $53.2_{-2.4}^{+3.2}$ & $0.72_{-0.05}^{+0.07}$ & $2.7_{-0.3}^{+0.4}$ & $3.7_{-0.5}^{+0.4} \times 10^{56}$ & $600_{-220}^{+150}$ & $0.12_{-0.04}^{+0.03}$ & 87 \\
\hline 0817 & $1.46_{-0.10}^{+0.12}$ & $1.27_{-0.09}^{+0.09}$ & $186_{-0.001}^{+0.001}$ & $0.00_{-0.01}^{+0.02}$ & $\leq 2.8$ & $\leq 0.89$ & $\geq 0.04$ & $\geq 0.1 \times 10^{56}$ & $40_{-15}^{+7}$ & $0.01_{-0.00}^{+0.00}$ & 16 \\
\hline GW170818 & $35.4_{-4.7}^{+7.5}$ & $26.7_{-5.2}^{+4.3}$ & $26.5_{-1.7}^{+2.1}$ & $-0.09_{-0.21}^{+0.18}$ & $59.4_{-3.8}^{+4.9}$ & $0.67_{-0.08}^{+0.07}$ & $2.7_{-0.5}^{+0.5}$ & $3.4_{-0.7}^{+0.5} \times 10^{56}$ & $1060_{-380}^{+420}$ & $0.21_{-0.07}^{+0.07}$ & 39 \\
\hline GW170823 & $39.5_{-6.7}^{+11.2}$ & $29.0_{-7.8}^{+6.7}$ & $29.2_{-3.6}^{+4.6}$ & $0.09_{-0.26}^{+0.22}$ & $65.4_{-7.4}^{+10.1}$ & $0.72_{-0.12}^{+0.09}$ & $3.3_{-0.9}^{+1.0}$ & $3.6_{-1.1}^{+0.7} \times 10^{56}$ & $1940_{-900}^{+970}$ & $0.35_{-0.15}^{+0.15}$ & 1666 \\
\hline
\end{tabular}

Table 1: Median values with $90 \%$ credible intervals for source parameters of the GWTC-1 detections. The columns report: component masses $\mathrm{m}_{1}, \mathrm{~m}_{2}$, chirp mass $\mathbf{M}_{c}$, dimensionless effective spin $\chi_{e f f}$, final source frame mass $\mathbf{M}_{f}$, final spin $\mathrm{a}_{f}$, radiated energy $\mathrm{E}_{r}$, peak luminosity $\mathrm{l}_{\text {peak }}$, luminosity distance $\mathrm{d}_{L}$, redshift $\mathrm{z}$, sky localization $\Delta \Omega$

mass $\mathrm{m}_{i},\left(G m_{i}^{2}\right) / c$, as $\vec{\chi}_{i}=\left(c \vec{S}_{i} /\left(G m_{i}^{2}\right)\right.$ and $a_{i}=\left(c\left|\vec{S}_{i}\right| /\left(G m_{i}^{2}\right)\right.$. The effective aligned spin is a combination of the spins weighted with masses and projected onto the Newtonian angular momentum $\hat{L}_{N}$ :

$$
\chi_{e f f}=\frac{\left(m_{1} \vec{\chi}_{1}+m_{2} \vec{\chi}_{2}\right) \cdot \hat{L}_{N}}{M}
$$

where $M=m_{1}+m_{2}$ is the total mass, with $m_{1} \geq m_{2}$. The merger of binary neutron stars needs an additional parameters to account for the tidal effects, the effective tidal deformability parameter $\tilde{\Lambda}$, related to the dimensionless tidal deformabilities of each neutron $\operatorname{star} \Lambda_{1}, \Lambda_{2}$, that in turn depend on their masses and equation of state [116], [247]:

$$
\tilde{\Lambda}=\frac{16}{13} \frac{\left(m_{1}+12 m_{2}\right) m_{1}^{4} \Lambda_{1}+\left(m_{2}+12 m_{1}\right) m_{2}^{4} \Lambda_{2}}{M^{5}}
$$

The estimated component masses of the binaries in the source frame are reported as contours in the $\mathrm{m}_{1}-\mathrm{m}_{2}$ plane in Fig. 2 for different values of the mass ratio $q=m_{2} / m_{1}$. The range of the black holes masses is consistent with the predictions for stellar mass black holes [72], [224]. The spins and the masses of the final black holes are reported in Fig. 2, right. The medians of all spin distributions cluster around 0.7 .

The spins of the initial compact systems have, in principle, arbitrary directions. The posterior distributions of the effective aligned spin parameter are shown in Fig. 3. The majority of posteriors are centered around zero, with the exception of the GW170729 and GW151226 events.

The luminosity distances of the binary black hole mergers range from 320 to $2840 \mathrm{Mpc}$ (redshift 0.49) [31], while GW170817 is at a distance of about $40 \mathrm{Mpc}$. The uncertainties on distances are relatively large, due to the degeneracy between the distance and the system inclination [97], [188], [115]. The mergers are localized via the arrival times and the waveform consistency at the interferometers [114], [132], [217]. The sky localization of all events is reported in Fig. 4, where the left panel shows the $\mathrm{O} 2$ events released to electromagnetic observers, while the right panels shows the $\mathrm{O} 1$ events and the unreleased $\mathrm{O} 2$ events. 


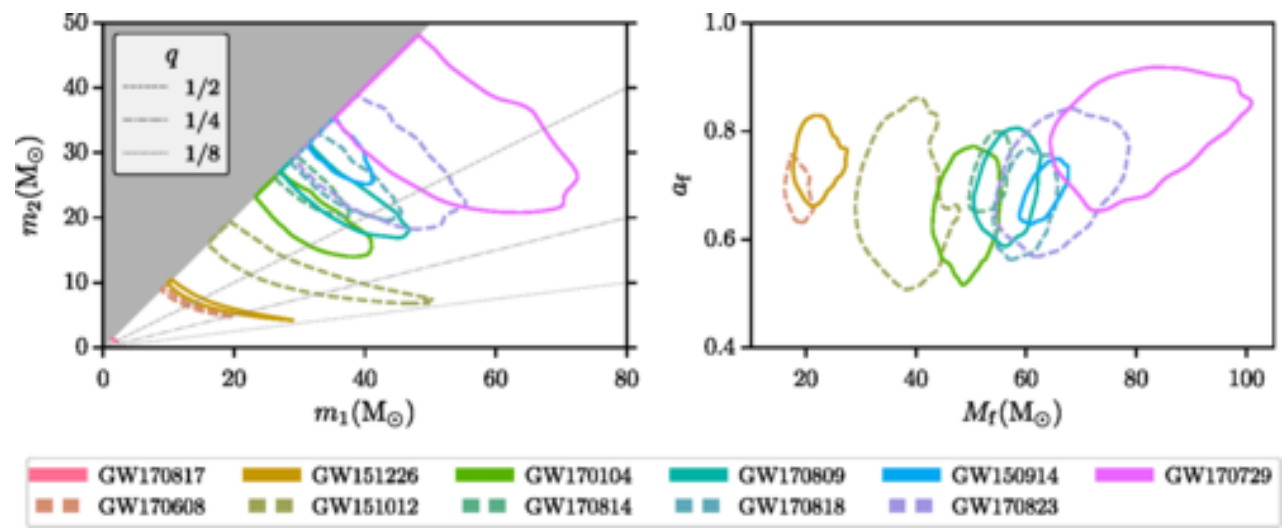

Figure 2: Posterior probability densities of the source frame component masses $\mathrm{m}_{1}, \mathrm{~m}_{2}$ (left) and of the mass $\mathbf{M}_{f}$ and dimensionless spin magnitude $\mathrm{a}_{f}$ of the final black hole (right); adapted from [31].

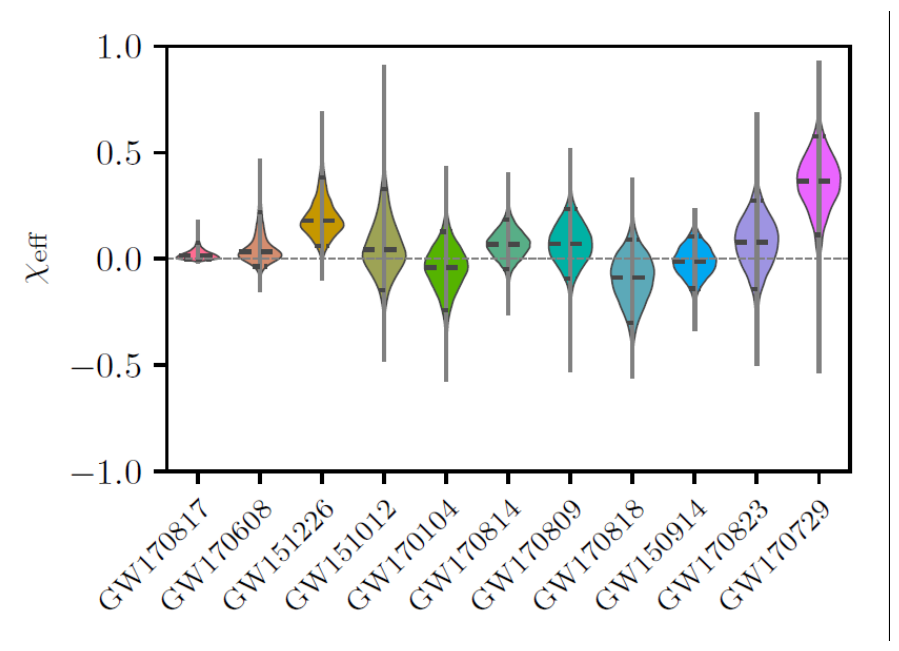

Figure 3: Estimated values of the effective aligned spin; adapted from [31].

The waveforms of all binary black hole events have been reconstructed with three different methods. Two of them are model independent methods: BAYESWAVE, that represents the waveform as a sum of sine-Gaussian wavelets [91]; coherent WaveBurst (cWB), that reconstructs the maximum likelihood signal [150]. The other method is Bayesian inference (LALINFERENCE), using the precessing waveform model PhenomP [240]. The time-frequency maps of the gravitational strain data measured in the interferometer with the higher SNR and the three reconstructions for all binary black hole mergers IN GWTC-1 catalog are shown in Fig. 5.

\section{GW170817}

On August 17, 2017 at 12:41:04 UTC the Advanced LIGO and Advanced Virgo interferometers detected the event GW170817 with a combined signal-to-noise ratio of 32.4 and an estimated false alarm rate smaller than one per $8.0 \times 10^{4}$ years [19]. The time frequency maps of the signals in the three interferometers are reported in Fig. 6, left. GW170817 was at a luminosity distance of 

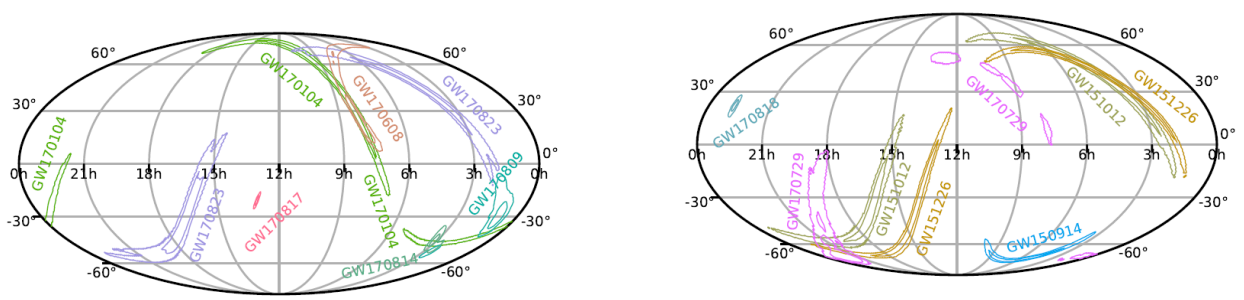

Figure 4: The 90\% and 50\% credible regions for the GWTC-1 events in a Mollweide projection; adapted from [31]. Left panel: O2 events GW170817, GW170104, GW170823, GW170608, GW170809, and GW170814 whose alerts were sent to astronomers; right panel: O1 events (GW150914, GW151226 and GW151012) and O2 events (GW170729 and GW170818) unreleased to astronomers.

$40_{-14}^{+8} \mathrm{Mpc}$ [19]. The observation of GW170817 with three interferometers localized GW170817 within about $28 \operatorname{deg}^{2}$ [20].

The estimated total mass ranges from 2.72 to $3.29 \mathrm{M}_{\odot}$, with individual masses from 0.86 to $2.26 \mathrm{M}_{\odot}$ [19], in agreement with the known masses of neutron stars in binary systems. The merger remnant [25], [33] could be either a neutron star or a black hole, that could produce gravitational waves with short $(\leq 1 \mathrm{~s})$ to intermediate $(\leq 500$ s) duration or $\mathrm{kHz}$ gravitational waves, respectively. The searches for signals of the final system have been negative [25], [33]. The progenitor of GW170817 has been discussed by [24].

\section{Follow-up of events in $\mathrm{O1}, \mathrm{O2}$}

Gravitational candidates are being monitored in the electromagnetic and neutrino domains, even when a counterpart is not expected, as in the case of binary black hole mergers. The follow up of gravitational events described in GWTC-1 catalog has been the subject of a large number of astronomical circulars and science papers. The searches for electromagnetic and neutrino counterparts of the $\mathrm{O} 1, \mathrm{O} 2$ events will be summarized here.

\subsection{Binary Black Hole Mergers in 01, $\mathrm{O2}$}

The preliminary estimations of time, significance and sky location of event GW150914 were shared with astronomer teams who covered the electromagnetic spectrum and neutrino domains with ground and space based observatories [12], in the first follow up of a gravitational event. GW150914 was observed in the optical domain by DECam [221], J-GEM [182], TOROS [103], iPTF [142], Pan-STARRS and PESSTO [219], MASTER [163], GRAWITA [83], TAROT [190] and in the radio domain with VLA [194]. X-ray observations were secured by INTEGRAL [209], Swift [112], XMM-Newton [231], AGILE [229], MAXI [146]. Gamma ray observations were performed with Fermi-LAT [44], InterPlanetary Network [139], Fermi-GBM [89]. All follow up observations were negative, with the exception of [89], that suggested the possible presence of a weak transient above $50 \mathrm{keV}, 0.4 \mathrm{~s}$ after the merger (see also [131], [90]). The neutrino searches with AUGER [1], ANTARES and IceCube [47], KamLAND [120], Super-Kamiokande [42] were also negative. The follow up of GW151226 included the optical observations by Pan-STARRS1 

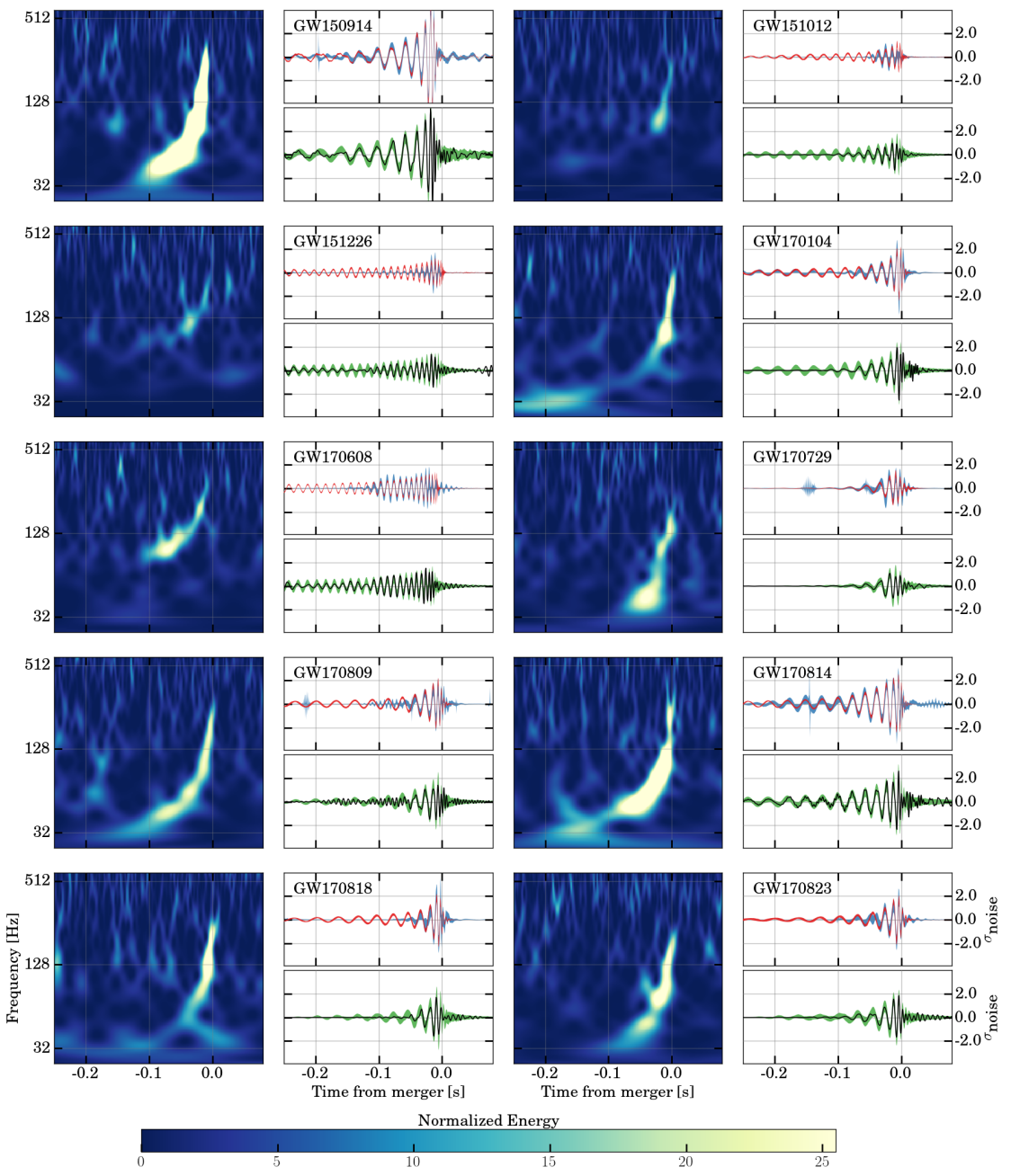

$\square$ BayesWave $\square$ LALInf $\square$ cWB-LALInf $\quad-$ cWB Max-L

Figure 5: Time-frequency graphs and reconstructed waveforms for the ten binary black hole mergers in GWTC-1 catalog. Each event is represented with three panels with the data of the LIGO interferometer with the higher SNR. The first panel shows the normalized time-frequency map of the strain; the other panels show the signal reconstructions in units of the noise standard deviation, for LALINFERENCE with the PhenomP (red) and BAYESWAVE wavelet models (blue) (top) and for cWB (bottom); adapted from [31]

[218], DECam [95], J-GEM [250], the radio VLA observations by [194], the gamma ray observations by CALET [48], [49] and Fermi-GBM, Fermi-LAT [202], the neutrino observations by AUGER [1], KamLAND [120], Super-Kamiokande [42], ANTARES and IceCube [53]. The follow up of GW151012 include Fermi-GBM and Fermi-LAT [202] and ANTARES and IceCube [53]. GW170104 was observed in the optical domain by TAROT [190], TOROS [64], DLT40 [249], in the radio domain [86], and at high energy with CALET [49], AGILE [242], INTEGRAL [211], Fermi-LAT and Fermi-GBM [124]. GW170608 was monitored in the optical domain by DLT40 [249], in the radio domain [63] and at high energies by CALET [49]. GW170814 was observed 

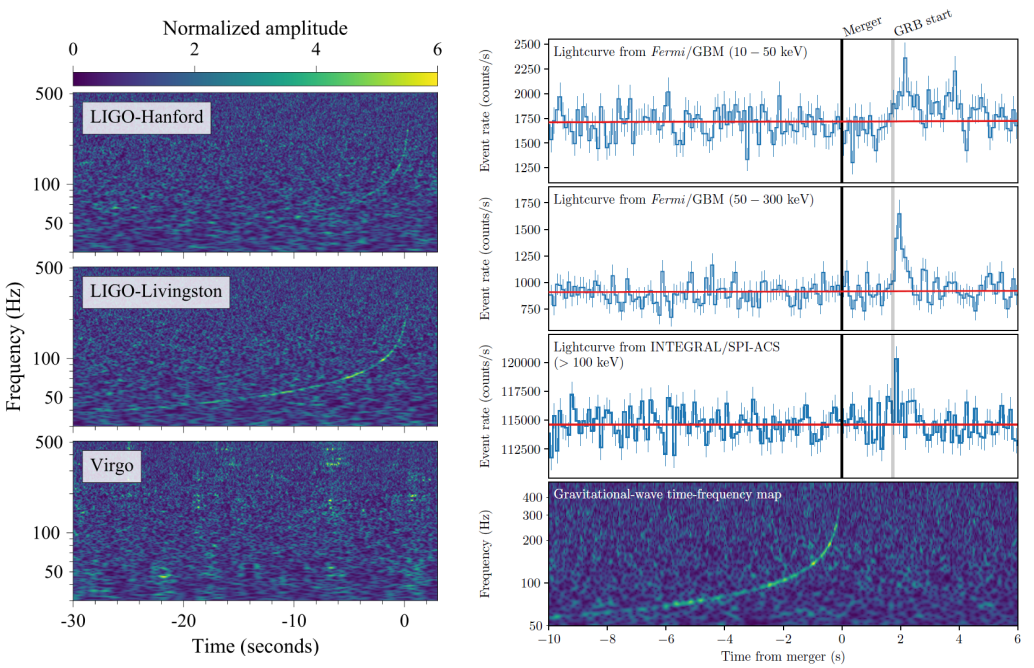

Figure 6: Left: time frequency maps of the GW170817 signal in the LIGO-Hanford, LIGO-Livingston and Virgo interferometers, adapted from [19]. Right: from top to bottom, joint detection of GW170817 and GRB 170817A by Fermi-GBM, 10-50 keV and 50-300 keV, INTEGRAL SPI-ACS (above $100 \mathrm{keV}$ ) and time-frequency map of GW170817 gravitational signal, adapted from [21]

in the optical domain by TAROT [190], the Dark Energy Camera [109], VLT [129], DLT40 [249] and at high energies with Swift-XRT [151] and CALET [49]. All searches for electromagnetic and neutrino counterparts of GW151226, GW151012, GW170104, GW170608, GW170814 were negative.

\subsection{GW170817}

GW170817 is the first detected merger of compact objects with a detected electromagnetic counterpart. The Fermi-GBM instrument observed the short Gamma Ray Burst GRB 170817A on 2017 August 17 at 12:41:06 UTC [123], [21], while the INTEGRAL instrument detected the event in an off-line analysis triggered by the LIGO-Virgo alert [210], [21]. The gravitational and gamma ray signals, shown in Fig. 6, right, showed a difference of $1.734 \pm 0.054 \mathrm{~s}$ in the arrival times [123], [21]. The prompt gamma ray emission has been discussed by [199]. No gamma ray excess was observed during the first days after the merger [241], [69], [49], [51], [170], [40], [41], [159].

The optical counterpart of GW170817, SSS17a/AT 2017gfo, was detected at 10.87 hours after the merger in the elliptical galaxy NGC 4993 [94], [215] and promptly confirmed [239], [227], [162]. [222]. [60], but was missing before the merger epoch [239]. The distance of NGC 4993 was consistent with the estimation of the gravitational luminosity distance. A worldwide network of observatories participated to the electromagnetic follow-up of SSS17a/AT 2017gfo [20], associated to GW170817 [24]. Optical and infrared photometric observations have been performed by several collaborations [238], [249], [61], [125], [104], [57], [85], [230], [62], [249], [64], see also the compilation by [244]. The optical spectra soon showed broad lanthanide features [214], [85], [171], [197], [165], [187] [143], [141], [148], [61], [96], [220], [110], [232], [157], [227], [88], as predicted by the kilonova model [192], [111], [193], [158], [152], [185], [70], [73], [226], [74], [101], [174], [245], [144]. 
After the first negative detections [225], [167], [210], [113], the X-ray afterglow of GW170817 was detected 9 days after the merger [232]. The radio afterglow of GW170817 was detected 16 days after the merger [135]. The optical afterglow was observed 109 days after the merger [165] and later [153], [119]. The X-ray and radio fluxes increased during the months after the merger [232], [133], [135], [55], [149], [178], [198], [205], [168], [203]. The initial brightness rise was followed by a peak and by a later decline [102], [107], [191], [179], [233], [84] [180],[122], [134], [235]. The compilation of optical, radio and X-ray data presented by [166] suggested that the peak occurred at 155 days after the merger (Fig. 7).

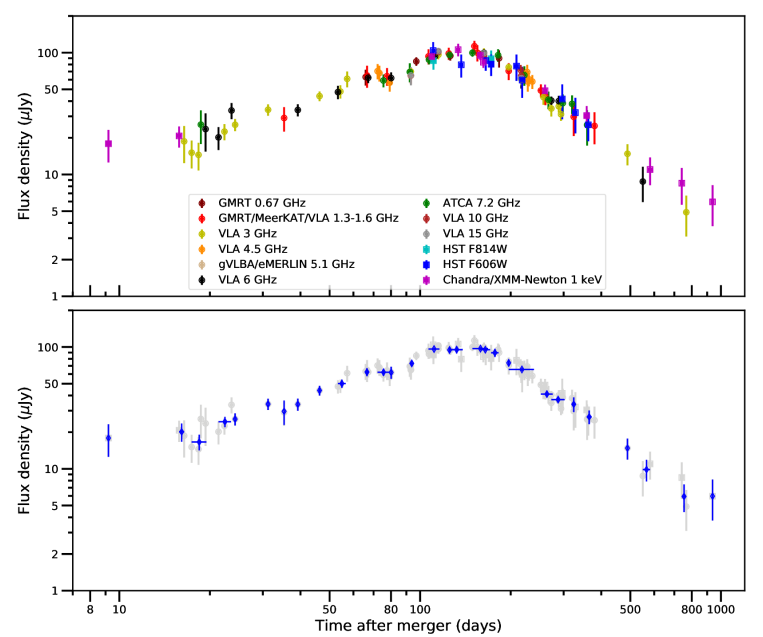

Figure 7: Upper panel: the panchromatic (optical, radio and X-ray) afterglow light curve of GW170817, from $+0.5 \mathrm{~d}$ to $+940 \mathrm{~d}$ after the merger. Lower panel: averaged light curve (blue data points). Credits: http://www.tauceti.caltech.edu/kunal/gw170817/

Neutrino observatories have searched for low and high energy neutrinos related to GW170817. All searches within a time window of $\pm 500 \mathrm{~s}$ and during 14 days after the merger have been negative [52], [43], [66], [196], [50].

\section{03 run}

The third LIGO-Virgo observing run (O3) started on April 1, 2019 and ended on 27 Mar 2020 due to COVID-19 pandemic. During the O3 run, event alerts have been public. The gravitational candidates were archived in the Gravitational Wave Candidate Event Database GraceDb ${ }^{1}$, while the alerts were distributed over the Gamma-Ray Coordinate Network $\mathrm{GCN}^{2}$. Run O3 produced 56 gravitational wave detections, well above the combined count of $\mathrm{O} 1$ and $\mathrm{O} 2$ runs (Fig. 8). Several groups have performed electromagnetic follow ups of events with a range of different instruments, among them MMT and SOAR [138], SAGUARO [164], GROWTH [93], GRANDMA [59], GOTO-4 [127].

On 2019 April 12 a binary black hole coalescence with asymmetric masses, GW190412, was observed [38], where a black hole with a mass of about $30 \mathrm{M}_{\odot}$ merged with a black hole of about

\footnotetext{
${ }^{1}$ https://gracedb.ligo.org

${ }^{2} \mathrm{https} / / / g \mathrm{gcn} . \mathrm{gsfc}$.nasa.gov/
} 


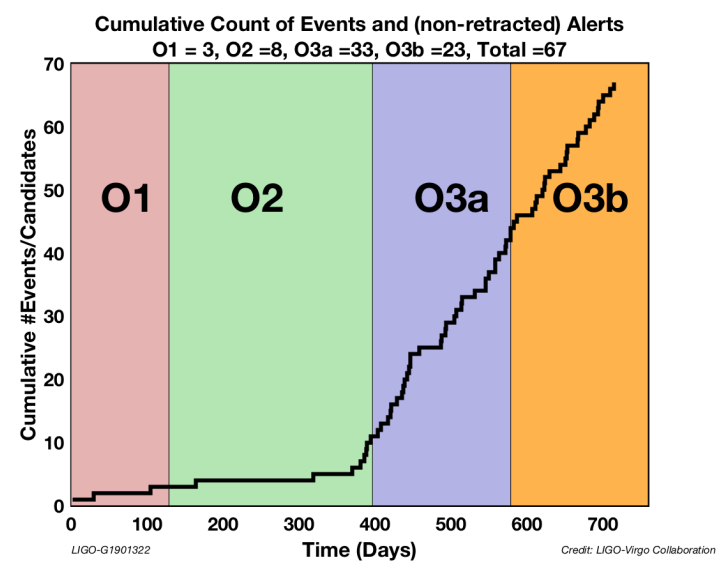

Figure 8: Cumulative counts of events in the O1, O2, O3 runs; image credit: LIGO-Virgo Collaboration.

$8 \mathrm{M}_{\odot}$. The more massive black hole had a dimensionless spin amplitude in the range between 0.17 and 0.59. There was evidence of gravitational emission beyond the main quadrupolar order, as expected for an asymmetric system [38].

On 2019 April 25 the merger GW190425 was detected [37]. The component masses range from 1.12 to $2.52 \mathrm{M}_{\odot}$, consistent with neutron stars as individual components. The total mass, $3.4_{-0.1}^{+0.3} \mathrm{M}_{\odot}$, and the source frame chirp mass, $1.44_{-0.02}^{+0.02} \mathrm{M}_{\odot}$, are larger than the corresponding values in known binary neutron star. Assuming that the event was a binary neutron star coalescence, the local rate of neutron star mergers was updated to $250-2810 \mathrm{Gpc}^{-3} \mathrm{yr}^{-1}$ [37]. There is no clear evidence for an electromagnetic counterpart [93], [138], [164], [121]. A weak Gamma Ray Burst, GRB 190425, was detected by the Anti-Coincidence Shield (ACS) of the SPI gamma-ray spectrometer onboard INTEGRAL [176], but not confirmed by Fermi-GBM [118] (see also [169], [212]). The time profile of GRB 190425 (two pulses at 0.5 and $5.9 \mathrm{~s}$ after the merger) is similar to that of GW170817 [200]. The absence of an optical counterpart could be explained by a large inclination of the jet axis with respect to the observer [200].

The event GW190814, detected on 2019 August 14, involved a 22.2-24.3 $\mathrm{M}_{\odot}$ black hole and a compact object with a mass of 2.50-2.67 $\mathrm{M}_{\odot}$ [39]. The merger was localized to $18.5 \mathrm{deg}^{2}$ at a distance of $241_{-45}^{+41} \mathrm{Mpc}$. The two primary black holes show the most unequal mass ratio yet observed with gravitational observations, about 0.11 , suggesting that the secondary component could be either the lightest black hole or the heaviest neutron star ever found in a compact object binary [39]. The estimated merger rate for this class of objects is $1-23 \mathrm{Gpc}^{-3} \mathrm{yr}^{-1}$ [39]. The optical follow up has been performed by [126], [58], [45], [248], [243], [181], while radio observations have been performed by [108]. No electromagnetic counterpart was found.

The event S190521g is a candidate black hole merger detected on 2019 May 21 at a luminosity distance of $3931 \pm 953 \mathrm{Mpc}$ and localized within a region of $765 \mathrm{deg}^{2}$ [160]. The transient ZTF19abanrhr, announced by the Zwycki Transient Facility at 34 days after the merger and linked with the active galactic nucleus J124942.3+344929 at a redshift of 0.438 , was associated with S190521g [130], the first candidate electromagnetic counterpart of a binary black hole merger [130]. The transient flare was consistent with a kicked binary black hole merger in the accretion disk of an active galactic nucleus, suggesting a total mass of about $100 \mathrm{M}_{\odot}$ [130]. A new en- 
counter of the source with the disk is expected to produce another flare in about 1.6 years [130]. The merging of compact objects in active galactic nuclei has been discussed by [71], [172].

\section{Physics and Astrophysics with Gravitational Waves}

The observation of gravitational waves from the binary black hole mergers GW150914 and GW151226 detected during the O1 run constrained the rate of binary black hole mergers in the range 9-240 $\mathrm{Gpc}^{-3} \mathrm{yr}^{-1}$ [10] and set upper limits on binary neutron star and black hole/neutron star mergers of $12600 \mathrm{Gpc}^{-3} \mathrm{yr}^{-1}$ and $3600 \mathrm{Gpc}^{-3} \mathrm{yr}^{-1}$, respectively [13]. The GWTC-1 catalog has updated the merger rates of compact binaries [31] as 9.7-101 $\mathrm{Gpc}^{-3} \mathrm{yr}^{-1}$ for binary black holes, 110-3840 $\mathrm{Gpc}^{-3} \mathrm{yr}^{-1}$ for binary neutron stars and set an upper limit of $610 \mathrm{Gpc}^{-3} \mathrm{yr}^{-1}$ for neutron star-black hole mergers.

The ten detections of the GWTC-1 catalog have constrained the properties of the black hole mass spectrum [34]. The mass distribution of the heavier black holes can be explained by models with a power law index of 1.3 [34]. The black hole binaries probably do not include black holes with large spins aligned to the orbital angular momentum [34]. The evolution of the merger rate is either flat or increasing with redshift [34].

The GWTC-1 catalog has been used for tests of General Relativity [32]. The measurements of the mass and the spin of the final black hole from the inspiral and the post-inspiral parts of the signal are in agreement [32]. The inspiral stage of the merger is described by the post-Newtonian (PN) formalism, that accounts for the most relevant effects of General Relativity [32]. The upper limit on the deviations from the post-Newtonian terms are shown in Fig. 9, left. Also the mergerringdown stages are in agreement with the predictions of General Relativity. Gravitational waves are not dispersed, according to General Relativity. The possible deviation is described by the amplitude A and the $\alpha$ parameter depending on the frequency of the gravitational waves. General Relativity predicts a value of zero for A; different $\alpha$ values are related to different theories alternative to General Relativity. The upper limit on the deviations from the dispersion relation predicted by General Relativity is shown in Fig. 9, right. The special case of $\alpha=0, \mathrm{~A}>0$ describes the propagation effects of a massive graviton, that is expected to be massless in General Relativity, but not in other alternative theories of gravity. The maximum limit on the graviton mass is $4.7 \times 10^{-23} \mathrm{eV} / \mathrm{c}^{2}$ [32].

The observation of GW170817 has constrained the physics of neutron stars: tidal deformability, radii, equations of state of neutron stars [19], [30], [26], [28], [31], [36]. The tidal deformability, that depends on the size and the equation of state of the neutron stars, has been constrained assuming that the two neutron stars have masses and spins consistent with those observed in known binary neutron stars and are described by the same equation of state [26]. The tidal deformabilities of the neutron stars involved in GW170817 are shown in Fig. 10. The radii of the neutrons star are in the range from 10.5 to $13.3 \mathrm{~km}[26]$.

The GW170817 detection has constrained the local coalescence rate of binary neutron star systems, $1540_{-1220}^{+3200} \mathrm{Gpc}^{-3} \mathrm{yr}^{-1}$ [19] and the contribution of unresolved systems to the stochastic gravitational wave background [27]. The first observation of an electromagnetic counterpart of the gravitational event GW170817 has constrained the fractional difference between the speed of light and the speed of gravity at the level of $10^{-15}-10^{-16}$ [21], using the delay of gamma rays with respect 

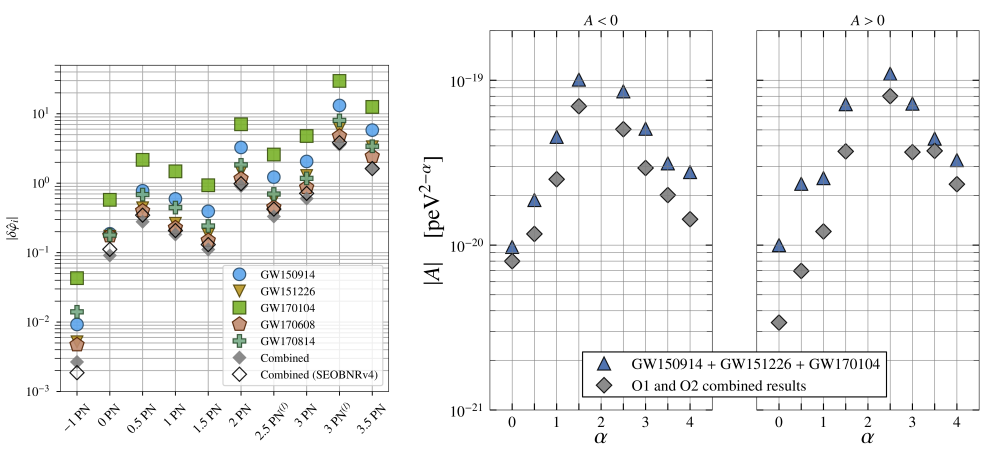

Figure 9: Left: upper limit on the deviations from the post-Newtonian terms describing the inspiral stage; adapted from [32]. Right: upper limit on the deviations from the dispersion relation (grey diamonds) and limits from previous gravitational events (blue triangles); adapted from [32]

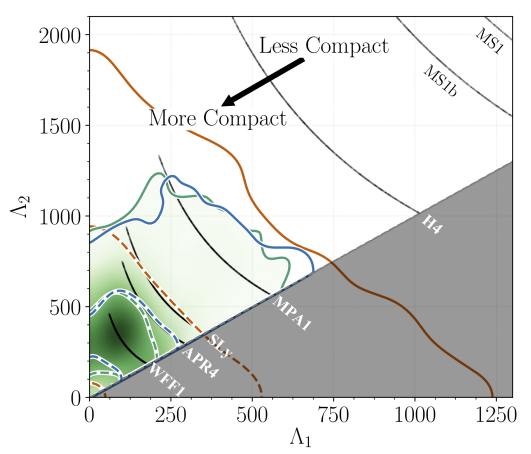

Figure 10: Tidal deformabilities of the two neutron stars in GW170817. The GW170817 system is within the green-shaded region, while the black lines are the predictions of some equations of state (adapted from [26])

to gravitational waves. A set of modified gravity model and dark energy models [56], [68], [154], [207] have been constrained or ruled out by the GW170817 observation. The multimessenger observations of GW170817 showed that the merger was followed by the short Gamma Ray Burst GRB170817A and by a kilonova, as predicted by [192], [111], [193], [158], [152], [185], [70], [73], [226], [74], [101], [174], [245], [144]. Binary neutron star mergers can explain short Gamma Ray Bursts or at least part of them. The mass distribution of observed ejecta in GW170817, 10 ${ }^{-3}$ to $10^{-2} \mathrm{M}_{\odot}$ [23] can explain the abundance of Galactic r-elements. The rising, the peak and the decline light curve of the GW170817 afterglow ruled out an on axis jet, supporting an off axis structured relativistic jet [113], [135], [54], [232], [167], [133], [149]. [183], [205], [165], [203], [156], [178], [155], [128], [92], [153], [168], [55], [180], [179], [145],[234], [119], [122], [134], [153], [186].

GW170817 has allowed, for the first time, tests of strong field dynamics of compact binaries in presence of matter [29]. The observation has constrained the dipole radiation, possible deviations from General Relativity in the post-Newtonian coefficients during the inspiral regime, modified dispersion of gravitational waves [29]. The combination of gravitational observations with electromagnetic observations has constrained effects from large extra dimensions [29] and supported 
evidence in favor of pure tensor polarization [29].

\section{Cosmology with Gravitational Waves}

GW170817 is the first detected standard siren, the gravitational version of the standard candle [213]. Presently, there is tension between the values of the Hubble constant estimated using CMB observations, $67.8 \pm 0.9 \mathrm{~km} \mathrm{~s}^{-1} \mathrm{Mpc}^{-1}$ [46] and the Cepheid variables, $73.48 \pm 1.66 \mathrm{~km} \mathrm{~s}^{-1} \mathrm{Mpc}^{-1}$ [204]. The luminosity distance of GW170817 combined with the recession velocity of the host galaxy NGC 4993 [22] has allowed an estimation of the Hubble constant, $70_{-8}^{+12} \mathrm{~km} \mathrm{~s} \mathrm{Mpc}^{-1}$, completely independent from the electromagnetic domain methods (Fig. 11). The precision on the Hubble constant is expected to improve at the level of $2 \%$ in five years and $1 \%$ in ten years with the detection of new binary neutron star merger events [87].

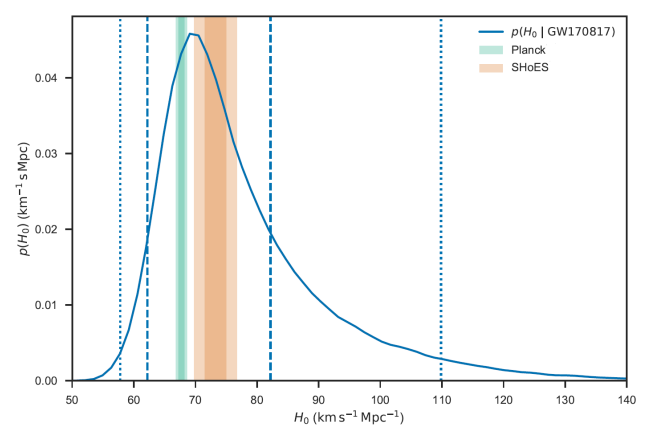

Figure 11: Relative probability of different values of Hubble constant estimated by [22] (solid blue curve), with limits of $68.3 \%$ and $95.4 \%$ credible intervals (dashed and dotted blue vertical lines); range of values estimated from CMB data, Planck satellite (green band) and from the SHoES analysis of Cepheids and type Ia supernovae (orange band). Adapted from [22]

The Hubble constant can be estimated also for events without electromagnetic counterpart using statistical methods involving the galaxies contained in the merger localization region [117], [223], [35]. A statistical analysis of GW170817 not using the knowledge of NGC 4993 as the host galaxy has been performed, considering all galaxies within the GW170817 localization region and combining their redshifts, leading to an estimate of the Hubble constant of $77_{-18}^{+37} \mathrm{~km} \mathrm{~s}^{-1} \mathrm{Mpc}^{-1}$ [117]. The binary black hole merger GW170814 has been used as a standard siren, combining its luminosity distance with the photometric redshifts of galaxies in the localization region from the catalog from the Dark Energy Survey (DES), giving a value of the Hubble constant of $75_{-32}^{+40} \mathrm{~km}$ $\mathrm{s}^{-1} \mathrm{Mpc}^{-1}$ [223]. The binary black hole detections from the $\mathrm{O} 1, \mathrm{O} 2$ runs have been used together with galaxy catalogs to estimate a value of the Hubble constant of $68_{-7}^{+14} \mathrm{~km} \mathrm{~s}^{-1} \mathrm{Mpc}^{-1}$ [35], with a leading role of the well localized GW170814 event. Several detections are expected over the next years, opening the way to cosmology with gravitational wave observations with and without electromagnetic counterparts.

\section{Conclusions}

The detection of binary black hole and binary neutron star mergers have started multi-messenger astronomy with gravitational waves. The events detected during the $\mathrm{O} 1, \mathrm{O} 2$ runs have produced 
the GWTC-1 catalog. The detections have an impact on astrophysics and cosmology, from the population of black holes to the physics of neutron stars to the estimation of the Hubble constant.

\section{References}

[1] A. Aab et al., PR D 94 (2016) 122007.

[2] B. P. Abbott et al., PRL 116 (2016) 061102.

[3] B. P. Abbott et al., PRL 116 (2016) 221101.

[4] B. P. Abbott et al., PRL 116 (2016) 241102.

[5] B. P. Abbott et al., PRL 116 (2016) 131102.

[6] B. P. Abbott et al., PR D 93 (2016) 122003.

[7] B. P. Abbott et al., PR D 93 (2016) 122004.

[8] B. P. Abbott et al., PR D 94 (2016) 064035.

[9] B. P. Abbott et al., $P R X$ 6 (2016) 041014.

[10] B. P. Abbott et al., PR X 6 (2016) 041015.

[11] B. P. Abbott et al., ApJL 818 (2016) L22.

[12] B. P. Abbott et al., ApJL 826 (2016) L13.

[13] B. P. Abbott et al., ApJL 832 (2016) L21.

[14] B. P. Abbott et al., ApJL 833 (2016) L1.

[15] B. P. Abbott et al., PRL 116 (2016) 241103.

[16] B. P. Abbott et al., PRL 118 (2017) 221101.

[17] B. P. Abbott et al., ApJ 851 (2017) L35.

[18] B. P. Abbott et al., PRL 119 (2017) 141101.

[19] B. P. Abbott et al., PRL 119 (2017) 161101.

[20] B. P. Abbott et al., ApJL 848 (2017) L12.

[21] B. P. Abbott et al., ApJL 848 (2017) L13.

[22] B. P. Abbott et al., Nat 551 (2017) 85.

[23] B. P. Abbott et al., ApJ 850 (2017) L39.

[24] B. P. Abbott et al., ApJ 850 (2017) L40.

[25] B. P. Abbott et al., ApJ 851 (2017) L16.

[26] B. P. Abbott et al., PRL 121 (2018) 161101.

[27] B. P. Abbott et al., PRL 120 (2018) 091101.

[28] B. P. Abbott et al., PRL 122 (2019) 061104.

[29] B. P. Abbott et al., PRL 123 (2019) 011102.

[30] B. P. Abbott et al., PRX 9 (2019) 011001. 
[31] B. P. Abbott et al., $P R X 9$ (2019) 031040.

[32] B. P. Abbott et al., PR D 100 (2019) 104036.

[33] B. P. Abbott et al., ApJ 875 (2019) 160.

[34] B. P. Abbott et al., ApJ 882 (2019) L24.

[35] B. P. Abbott et al., arXiv:1908.06060.

[36] B. P. Abbott et al., CQG 37 (2020) 045006.

[37] B. P. Abbott et al., ApJL 892 (2020) L3.

[38] R. Abbott et al., eprint arXiv:2004.08342.

[39] R. Abbott et al., ApJL 896 (2020) L44.

[40] H. Abdalla et al., ApJ 850 (2017) 22.

[41] H. Abdalla et al., ApJL 894 (2020) L16.

[42] K. Abe et al., ApJL 830 (2016) L11.

[43] K. Abe et al., ApJ 857 (2018) L4.

[44] M. Ackermann et al., ApJL 823 (2016) L2.

[45] K. Ackley et al., eprint arXiv:2002.01950.

[46] P. A. R. Ade et al., $A \& A 594$ (2016) A13.

[47] S. Adrian-Martinez et al., PR D 93 (2016) 122010.

[48] O. Adriani et al., ApJL 829 (2016) L20.

[49] O. Adriani et al., ApJ 863 (2018) 160.

[50] N. Y. Agafonova et al., J. Phys.: Conf. Ser. 1390 (2019) 012088.

[51] N. Ajello et al., ApJ 861 (2018) 85.

[52] A. Albert et al., ApJ 850 (2017) L35.

[53] A. Albert et al., PR D 96 (2017) 022005.

[54] K. D. Alexander et al., ApJL 848 (2017) L21.

[55] K. D. Alexander et al., ApJ 863 (2018) L18.

[56] L. Amendola et al., PRL 120 (2018) 131101.

[57] I. Andreoni et al., PASA 34 (2017) 169.

[58] I. Andreoni et al., ApJ 890 (2020) 131.

[59] S. Antier et al., MNRAS 492 (2020) 3904.

[60] I. Arcavi et al., Nat 551 (2017) 64.

[61] I. Arcavi et al., ApJL 848 (2017) L33.

[62] I. Arcavi et al., ApJ 855 (2018) L23.

[63] K. Artkop et al., ApJ 884 (2019) 16.

[64] R. Artola et al., MNRAS 493 (2020) 2207. 
[65] K. G. Arun et al., PR D 79 (2009) 104023.

[66] A. D. Avrorin et al., JETPL 108 (2018) 787.

[67] S. Babak et al., PR D 95 (2017) 024010.

[68] T. Baker et al., PRL 119 (2017) 251301.

[69] A. Balasubramanian et al., GCN 21514 (2017).

[70] J. Barnes and D. Kasen, ApJ 775 (2013) 18.

[71] I. Bartos et al., ApJ 835 (2020) 165.

[72] K. Belczynski et al., ApJ 714 (2010) 1217.

[73] E. Berger et al., ApJL 774 (2013) L13.

[74] E. Berger, ARA\&A 52 (2014) 43.

[75] S. Bernuzzi et al., PRL 114 (2015) 161103.

[76] L. Blanchet et al., PRL 74 (1995) 3515.

[77] L. Blanchet et al., PRL 93 (2004) 091101.

[78] L. Blanchet et al., PR D 71 (2005) 124004.

[79] L. Blanchet, LRR 17 (2014) 2.

[80] A. Bohé et al., CQG 30 (2013) 135009.

[81] A. Bohé et 1., CQG 32 (2015) 195010.

[82] A. Bohé et al., PR D 95 (2017) 044028.

[83] E. Brocato et al., MNRAS 474 (2018) 411.

[84] J. W. Broderick et al., MNRAS 494 (2020) 5110.

[85] D. A. H. Buckley et al., MNRAS 474 (2018) L71.

[86] T. Callister et al., ApJL 877 (2019) L39.

[87] H.-Y. Chen et al., Nat 562 (2018) 545.

[88] R. Chornock et al., ApJ 848 (2017) L19.

[89] V. Connaughton et al., ApJL 826 (2016) L6.

[90] V. Connaughton et al., ApJL 853 (2018) L9.

[91] N. J. Cornish and T. B. Littenberg, CQG 32 (2015) 135012.

[92] A. Corsi et al., ApJ 861 (2018) L10.

[93] M. W. Coughlin et al., ApJL 885 (2019) L19.

[94] D. A. Coulter et al., Sci 358 (2017) 1556.

[95] P. S. Cowperthwaite et al., ApJL 826 (2016) L29.

[96] P. S. Cowperthwaite et al., ApJ 848 (2017) L17.

[97] C. Cutler and E. E. Flanagan, PR D 49 (1994) 2658.

[98] T. Damour et al., PL B 513 (2001) 147. 
[99] T. Damour and A. Nagar, $P R$ D 81 (2010) 084016.

[100] T. Damour et al., PR D 85 (2012) 123007.

[101] P. D'Avanzo, JHEAp 7 (2015) 73.

[102] P. D'Avanzo et al., $A \& A 613$ (2018) L1.

[103] M. C. Diaz et al., ApJL 828 (2016) L16.

[104] M. C. Diaz et al., ApJL 848 (2017) L29.

[105] T. Dietrich et al., $P R D 96$ (2017) 121501.

[106] T. Dietrich et al., PR D 99 (2019) 024029.

[107] D. Dobie et al., ApJ 858 (2018) L15.

[108] D. Dobie et al.,ApJL 887 (2019) L13.

[109] Z. Doctor et al., ApJL 873 (2019) L24.

[110] M. R. Drout et al., Sci 358 (2017) 1570.

[111] D. Eichler et al., Nat 340 (1989) 126.

[112] P. A. Evans et al., MNRAS 460 (2016) L40.

[113] P. A. Evans et al., Sci 358 (2017) 1565.

[114] S. Fairhurst, NJ 11 (2009).

[115] B. Farr et al., ApJ 825 (2016) 116.

[116] M. Favata, PRL 112 (2014) 101101.

[117] M. Fishbach et al., ApJ 871 (2019) L13.

[118] C. Fletecher et al., GCN 24185 (2019).

[119] W. Fong et al., ApJL 883 (2019) L1.

[120] A. Gando et al., ApJL 829 (2016) L34.

[121] https://gcn.gsfc.nasa.gov/other/S190425z.gcn3.

[122] G. Ghirlanda et al., Sci 363 (2019) 968.

[123] A. Goldstein et al., ApJ 848 (2017) L14.

[124] A. Goldstein et al., ApJL 846 (2017) L5.

[125] V. Z. Golkhou et al., ApJ 857 (2018) 81.

[126] S. Gomez et al., ApJL 884 (2019) L55.

[127] B. P. Gompertz et al., arXiv:2004.00025.

[128] O. Gottlieb et al., MNRAS 473 (2018) 576.

[129] A. Grado et al., MNRAS 492 (2020) 1731.

[130] M. J. Graham et al., PRL 124 (202) 251102.

[131] J. Greiner et al., ApJL 827 (2016) L38.

[132] K. Grover et al., PR D 89 (2014) 042004. 
[133] D. Haggard et al., ApJL 848 (2017) L25.

[134] A Hajela et al., ApJ 886 (2019) L17.

[135] G. Hallinan et al., Sci 358 (2017) 1579.

[136] M. Hannam et al., PRL 113 (2014) 151101.

[137] T. Hinderer et al.,PRL 116 (2016) 181101.

[138] G. Hosseinzadeh et al., ApJL 880 (2019) L4. (2019).

[139] K. Hurley et al., ApJL 829 (2016) L12.

[140] S. Husa et al., PR D 93 (2016) 044006.

[141] D. Kasen et al., Nat 551 (2017) 80.

[142] M. M. Kasliwal et al., ApJL 824 (2016) L24.

[143] M. M. Kasliwal et al., Sci 358 (2017) 6370.

[144] M. M. Kasliwal et al.,MNRAS, arXiv:1812.08708 (2019).

[145] A. Kathirgamaraju et al., MNRAS 473 (2018) L121.

[146] N. Kawai et al., PASJ 69 (2017) 84.

[147] S. Khan et al., PR D 93 (2016) 044007.

[148] C. D. Kilpatrick et al., Sci 358 (2017) 1583.

[149] S. Kim et al., ApJ 850 (2017) 850.

[150] S. Klimenko et al., PR D 93 (2016) 042004.

[151] N. J. Klingler et al., ApJSS 245 (2019) 15.

[152] S. Kulkarni, arXiv: astro-ph/0510256 (2005).

[153] G. P. Lamb et al., ApJ 870 (2019) 15.

[154] D. Langlois et al., PRD 97 (2018) 061501.

[155] D. Lazzati et al., ApJL 848 (2017) L6.

[156] D. Lazzati et al., PRL 120 (2018) 241103.

[157] A. J. Levan et al., ApJL 848 (2017) L28.

[158] L.-Z. Li and B. Paczynski, ApJ 507 (1998) 59.

[159] T. Li et al., SCPMA 61 (2018) 31011.

[160] Ligo Scientific Collaboration; VIRGO Collaboration, GCN Circular 24621 (2019).

[161] V. M. Lipunov et al., MNRAS 288 (1997) 245.

[162] V. M. Lipunov et al., ApJL 850 (2017) L18.

[163] V. M. Lipunov et al., MNRAS 465 (2017) 3656.

[164] M. J. Lundquist et al., ApJL 881 (2019) L26.

[165] J. D. Lyman et al., NatAs 2 (2018) 751.

[166] S. Makhathini et al., arXiv:2006.02382. 
[167] R. Margutti et al., ApJL 848 (2017) L20.

[168] R. Margutti et al., ApJ 856 (2018) L18.

[169] A. Martin-Carillo et al., GCN 24169 (2019).

[170] I. Martinez-Castellanos et al., GCN 21683 (2017).

[171] C. McCully et al., ApJL 848 (2017) L32.

[172] B. McKernan et al., MNRAS 494 (2020) 1203.

[173] C. Messick et al., PRD 95 (2017) 042001.

[174] B. D. Metzger, LRR 20 (2017) 3.

[175] B. Mikoczi et al,, PR D 71 (2005) 124043.

[176] P. Minaev et al., GCN 24170 (2019).

[177] C. K. Mishra et al., PR D 93 (2016) 084054.

[178] K. P. Mooley et al., Nat 554 (2018) 207.

[179] K. P. Mooley et al., ApJ 868 (2018) L11.

[180] K. P. Mooley et al., Nat 561 (2018) 355.

[181] R. Morgan et al., eprint arXiv:2006.07385.

[182] T. Morokuma et al., PASJ 68 (2016) L9.

[183] A. Murguia-Berthier et al., ApJL 848 (2017) L34.

[184] A. Nagar et al., PR D 98 (2018) 104052.

[185] E. Nakar, PhR 442 (2007) 166.

[186] E. Nakar and T. Piran, MNRAS 478 (2018) 407.

[187] M. Nicholl et al., ApJ 848 (2017) L18.

[188] S. Nissanke et al., ApJ 725 (2010) 496.

[189] A. H. Nitz et al.,PyCBC Software, http://github .com/ligo-cbc/pycbc.

[190] K. Noysena et al., ApJ 886 (2019) 73.

[191] M. Nynka et al., ApJL 862 L19. (2018).

[192] B. Paczynski, ApJL 308 (1986) L43.

[193] B. Paczynski, AcA 41 (1991) 257.

[194] N. T. Palliyaguru et al., ApJL 829 (2016) L28.

[195] Y. Pan et al., PR D 89 (2014) 084006.

[196] V. B. Petkov et al., JETP Lett. 107 (2018) 398.

[197] E. Pian et al., Nat 551 (2017) 67.

[198] D. Pooley et al., ApJL 859 (2018) L23.

[199] A. S. Pozanenko et al., ApJ 852 (2018) L30.

[200] A. S. Pozanenko et al., AstL 45 (2020) 710. 
[201] M. Pürrer, CQG 31 (2014) 195010.

[202] J. L. Racusin et al., ApJ 835 (2017) 82.

[203] L. Resmi et al., ApJ 867 (2018) 57.

[204] A. G. Riess et al., ApJ 855 (2018) 136.

[205] J. R. Ruan et al., ApJ 853 (2018) L4.

[206] S. Sachdev et al., arXiv:1901.08580.

[207] R. H. Sanders, IJMPD 27 (2018) 1847027.

[208] B. S. Sathyaprakash and S. V. Dhurandhar, PR D 44 (1991) 381.

[209] V. Savchencko et al., ApJL 820 (2016) L36.

[210] V. Savchenko et al., ApJ 848 (2017) L15.

[211] V. Savchencko et al., ApJL 846 (2017) L23.

[212] V. Savchencko et al., GCN 24178 (2019).

[213] B. F. Schutz, Nat 323 (1986) 310.

[214] B. J. Shappee et al., Sci 358 (2017) 1574.

[215] M. R. Siebert et al., ApJL 848 (2017) L26.

[216] S. Sigurdsson and L. Hernquist, Nat 364 (1993) 423.

[217] L. P. Singer and L. R. Price. PR D 93 (2016) 024013.

[218] S. J. Smartt et al., ApJL 827 (2016) L40.

[219] S. J. Smartt et al., MNRAS 462 (2016) 4094.

[220] S. J. Smartt et al., Nat 551 (2017) 75.

[221] M. Soares-Santos et al., ApJL 823 (2016) L33.

[222] M. Soares-Santos et al., ApJL 848 (2017) L16.

[223] M. Soares-Santos et al., ApJL 876 (2019) L7.

[224] M. Spera et al., MNRAS 451 (2015) 4086.

[225] S. Sugita et al., PASJ 70 (2018) 81.

[226] N. R. Tanvir et al., Nat 500 (2013) 547.

[227] N. R. Tanvir et al., ApJL 848 (2017) L27.

[228] A. Taracchini et al., PR D 89 (2014) 061502.

[229] M. Tavani et al., ApJL 825 (2016) L4.

[230] N. Tominaga et al., PASJ 70 (2018) 28.

[231] E. Troja et al., ApJL 822 (2016) L8.

[232] E. Troja et al., Nat 551 (2017) 71.

[233] E. Troja et al., MNRAS 478 (2018) L18.

[234] E. Troja et al., MNRAS 489 (2019) 1919. 
[235] E. Troja et al., arXiv:2006.01150.

[236] A. Tutukov and L. Yungelson, NInfo 27 (1973) 70.

[237] S. A. Usman et al., CQG 33 (2016) 215004.

[238] Y. Utsumi et al., PASJ 69 (2017) 101.

[239] S. Valenti et al., ApJL 848 (2017) L24.

[240] J. Veitch et al., PR D 91 (2015) 042003.

[241] F. Verrecchia et al., ApJ 850 (2017) L27.

[242] S. Verrecchia et al., ApJL 847 (2017) L20.

[243] N. Vieira et al., ApJ 895 (2020) 96.

[244] V. A. Villar et al., ApJL 851 (2017) L21.

[245] V. A. Villar et al., ApJ 862 (2018) L11.

[246] J. Vines et al., $P R D \mathbf{8 3}$ (2011) 084051.

[247] L. Wade et al., PR D 89 (2014) 103012.

[248] A. M. Watson et al., MNRAS 492 (2020) 5916.

[249] S. Yang et al., ApJ 875 (2019) 59.

[250] M. Yoshida et al., PASJ 69 (2017) 9. 